\title{
Analysis of the Electromagnetic Inductive Response of a Void in a Conducting-Soil Background
}

\author{
Tiejun Yu, Member, IEEE, and Lawrence Carin, Senior Member, IEEE
}

\begin{abstract}
A lossless dielectric object situated in a lossy dielectric medium (soil) constitutes a void in a conducting background, which can be detected via an electromagnetic-induction (EMI) sensor operating at appropriate frequencies. The electromagnetic character of this void is dependent on the target and soil properties, as well as on the frequency of operation. We utilize the rigorous method of moments (MoM) and the approximate extended-Born technique to model this three-dimensional (3-D) problem. The modeling algorithms are discussed in detail, with a focus on efficient computation of the dyadic Green's function at the frequencies of interest. The MoM results are used to calibrate the accuracy of the approximate extended-Born solution, over a wide range of operating conditions. Furthermore, the computer simulations are used to perform a detailed phenomenological study.
\end{abstract}

Index Terms-Electromagnetic induction, extended-Born, scattering.

\section{INTRODUCTION}

$\mathbf{T}$ HERE are many applications for which one may be interested in sensing a low-loss dielectric target embedded in a lossy host medium. For example, buried plastic land mines [1]-[4], plastic pipes [5], and underground tunnels generally constitute voids in the presence of a lossy soil background. While such targets can in principle be detected by a radar sensor [1]-[5], radar suffers well-known difficulties due to soil-induced attenuation [1]. An electromagnetic-induction (EMI) sensor [6], [7] on the other hand, affords the potential for significant soil penetration. Such sensors are typically used as metal detectors, with example applications including detection of buried conducting land mines and unexploded ordnance [8]-[10]. As elucidated here, such sensors can also be used to detect the absence of conductivity (a void) in the presence of a conducting host medium. Our focus here is on generally small, shallow targets (e.g., plastic mines), although the computer model is quite general. For such a problem, while the target's electrical properties cannot be changed, those of the host soil can. We therefore address the character of the EMI conductivity-void signature as a function of soil water content. The objective is to increase the soil conductivity by increasing the soil water content [1], thereby enhancing the conductivity contrast between the plastic target and soil, yielding an increased target signature. Clearly, many types of clutter may also

\footnotetext{
Manuscript received June 4, 1999; revised November 30, 1999. This work was supported in part by the U.S. Joint Unexploded Ordnance Coordination Office, Adelphi, MD, and in part by the DoD Multidisciplinary University Research Initiative (MURI) in demining.

The authors are with Department of Electrical and Computer Engineering, Duke University, Durham, NC 27708-0291 USA.

Publisher Item Identifier S 0196-2892(00)03926-7.
}

manifest a void in a conducting background (e.g., tree roots, rocks, etc.). Therefore, the objective is to use the EMI-based void detector as a queuing device for other devices such as chemical sensors [11], these also being applicable to land mines with low or no metal content. Furthermore, although not pursued here, our forward model could be applied in the context of inverse scattering [6] to yield improved information on the details of the subsurface target (void).

The rigorous modeling of EMI interaction with a buried void in a conducting host medium presents several challenges. Focusing on a frequency-domain, integral-equation analysis [2], [12]-[15], one must carefully evaluate the dyadic layered-medium Green's function [2], [12]-[15] for a layered-medium soil model. For radar-based problems, the method of complex images [2], [14], [15] has proven a very efficient and accurate tool for the analysis of the requisite Sommerfeld integrals [2], [12]-[15]. However, we have found this technique less useful for the EMI frequencies of interest here. Consequently, we have employed efficient interpolation-based Green's-function computation, similar in spirit to that presented in [12].

Having realized an efficient and accurate technique for Green's-function computation, we are still left with the problem of solving the integral equation. We have here applied a volumetric electric field integral equation (EFIE) [12], in which the unknowns are the total electric fields inside the dielectric target for a known heterogeneity between the target and the background (soil) medium [12]. Such an EFIE is often solved via a volumetric method of moments (MoM) solution [12]. While a MoM analysis is accurate, it generally requires the inversion of a large matrix. This task is circumvented by employing the extended-Born method [16], [17]. This is an approximate method that only requires inversion of simple $3 \times 3$ matrices. The attendant speed enhancement accrued by extended-Born, vis- $a$-vis the MoM, is critical if the model is to be utilized in the context of signal processing and/or inverse scattering [18]. An example of employing sophisticated scattering models in the context of target detection and classification can be found in [1].

While the reduced computational complexity of extended-Born is attractive, it is important that the results not suffer markedly in accuracy. Therefore, in the work reported here, we perform a detailed frequency-dependent comparison between the rigorous MoM and approximate extended-Born solutions. This test is performed using parameters from an actual EMI sensor [7] for several different target and soil scenarios. In the context of these comparisons, we also investigate the phenomenology underlying EMI-based buried-void detection. 
The remainder of the paper is organized as follows. In Section II, we review the electric-field integral equation characteristic of the buried-void problem. We discuss the integral-equation solution in the context of both the rigorous MoM and the approximate extended-Born methods. In this section, we also elucidate the technique applied for Green's-function evaluation. A detailed comparison is performed in Section III between the MoM and extended-Born solutions, wherein we also address the associated wave phenomenology. Finally, conclusions are addressed in Section IV.

\section{THEORY}

\section{A. Electric Field Integral Equation and MoM Solution}

The volumetric EFIE for the layered-earth model has been derived in [12], and therefore here is only provided a summary. Assume that $\boldsymbol{E}(\boldsymbol{r})$ represents the electric field at the position $\mathrm{r}$ and that $\boldsymbol{E}^{\text {inc }}(\boldsymbol{r})$ represents the incident fields produced by the excitation in the absence of the target. Using $\boldsymbol{G}\left(\boldsymbol{r}, \boldsymbol{r}^{\prime}\right)$ to denote the dyadic, layered-medium Green's function, we have [12]

$\boldsymbol{E}(\boldsymbol{r})=\boldsymbol{E}^{i n c}(\boldsymbol{r})-j \omega \int_{V}\left[\varepsilon_{t}\left(\boldsymbol{r}^{\prime}\right)-\varepsilon_{b}\left(\boldsymbol{r}^{\prime}\right)\right] G\left(\boldsymbol{r}, \boldsymbol{r}^{\prime}\right) \cdot \boldsymbol{E}\left(\boldsymbol{r}^{\prime}\right) d^{3} \boldsymbol{r}^{\prime}$

where an $\exp (j \omega t)$ time dependence is assumed and suppressed. In (1), $\varepsilon_{b}$ represents the inhomogeneous dielectric constant of the background layered medium, and $\varepsilon_{t}$ represents the dielectric constant of the generally inhomogeneous target, with the latter assumed (for numerical simplicity) to reside entirely within a single layer of $\varepsilon_{b}$. In general, the layered background medium and the target are lossy and therefore, both $\varepsilon_{b}$ and $\varepsilon_{t}$ are complex [19].

For a known excitation $\boldsymbol{E}^{\boldsymbol{i n c}}(\boldsymbol{r})$, (1) implies that knowledge of the total electric field inside the target can be used to calculate $\boldsymbol{E}(\boldsymbol{r})$ everywhere. Therefore, the problem reduces to solving for the total electric field $\boldsymbol{E}(\boldsymbol{r})$ for $\boldsymbol{r}$ inside the target volume $V$. In the MoM solution, we expand the unknown $\boldsymbol{E}(\boldsymbol{r})$ for $\boldsymbol{r} \in V$ in terms of known basis functions with unknown coefficients

$$
\boldsymbol{E}(\boldsymbol{r})=\boldsymbol{x} \sum_{n=1}^{N} a_{n} e_{x n}(\boldsymbol{r})+\boldsymbol{y} \sum_{n=1}^{N} b_{n} e_{y n}(\boldsymbol{r})+z \sum_{n=1}^{N} c_{n} e_{z n}(\boldsymbol{r})
$$

where

$$
\begin{array}{ll}
\boldsymbol{x}, \boldsymbol{y} \text { and } z & \text { unit vectors in Cartesian coordinates; } \\
a_{n}, b_{n}, \text { and } c_{n} & \text { unknown basis-function coefficients; } \\
e_{x n}, e_{y n}, \text { and } & \text { known basis functions for the electric field } \\
e_{z n} & \text { in the } x, y \text { and } z \text { directions, respectively. }
\end{array}
$$

As in [12], we utilize simple cube expansion functions for all $e_{x n}, e_{y n}$ and $e_{z n}$.

Employing (2) inside the integral in (1) and applying point matching [20] inside each of the $3 N$ basis-functions (cubes), we attain the MoM matrix equation

$$
Z i=v
$$

where

$Z 3 N \times 3 N$ MoM "impedance" matrix;

$i \quad 3 N \times 1$ vector representing the unknown coefficients $a_{n}, b_{n}$, and $c_{n}$; $v \quad 3 N \times 1$ vector representing the point-matched incident electric field (at the center of each cubic basis function).

One can use (3) to solve for the unknown $i$, this constituting the procedure applied in [12]. The MoM, representing a rigorous analysis, constitutes a reference solution. However, we are also interested in developing a more efficient, albeit approximate, solution. This is motivated by the fact that an LU-decomposition solution of (3) is of order $N^{3}$ complexity, while an iterative, conjugate-gradient (CG) solution [21] has complexity of order $P N^{2}$, where $P$ is the number of CG iterations ( $P$ may approach $N$ if a good preconditioner is not used). For large $N$, such computations can become prohibitive. Independent of the matrix solver, order $N^{2}$ complexity is required to fill the matrix $Z$. Some of the complexity of the MoM solution can be reduced by exploiting convolutional properties of the integral equation [22] through use of the fast Fourier transform (FFT).

\section{B. Extended-Born Solution}

The extended-Born method is an approximate technique first developed in [16], [17]. Here we extend this algorithm to threedimensional layered-media, for a realistic excitation. The algorithm is summarized as follows. The "extended-Born" approximation [16], [17] is based on recognizing that $\boldsymbol{G}\left(\boldsymbol{r}, \boldsymbol{r}^{\prime}\right)$ is large for $\boldsymbol{r}$ in the vicinity of $\boldsymbol{r}^{\prime}$, while being relatively small for $\boldsymbol{r}$ distant from $\boldsymbol{r}^{\prime}$. Consequently, from (1) we have

$$
\begin{gathered}
\boldsymbol{E}(\boldsymbol{r}) \approx \boldsymbol{E}^{i n c}(\boldsymbol{r})-j \omega \int_{V}\left[\varepsilon_{t}\left(\boldsymbol{r}^{\prime}\right)-\varepsilon_{b}\left(\boldsymbol{r}^{\prime}\right)\right] \boldsymbol{G}\left(\boldsymbol{r}, \boldsymbol{r}^{\prime}\right) \cdot \boldsymbol{E}(\boldsymbol{r}) d v^{\prime} \\
\text { for } \boldsymbol{r} \in V .
\end{gathered}
$$

Rearranging (4), we find

$$
\begin{aligned}
\boldsymbol{E}(\boldsymbol{r}) & \approx \boldsymbol{M}(\boldsymbol{r})^{-1} \cdot \boldsymbol{E}^{i n c}(\boldsymbol{r}) \quad \text { for } \boldsymbol{r} \in V \\
\boldsymbol{M}(\boldsymbol{r}) & \equiv \boldsymbol{I}+j \omega \int_{V} \boldsymbol{G}\left(\boldsymbol{r}, \boldsymbol{r}^{\prime}\right)\left[\varepsilon\left(\boldsymbol{r}^{\prime}\right)-\varepsilon_{t}\left(\boldsymbol{r}^{\prime}\right)\right] d^{3} \boldsymbol{r}^{\prime}
\end{aligned}
$$

where $\boldsymbol{I}$ is a $3 \times 3$ diagonal matrix with $\boldsymbol{I}_{k k}=1$. The expression in (5) yields an approximation for the electric fields inside the target, which can be used in (1) to find the fields everywhere (i.e., for $\boldsymbol{r} \notin V$ ). Note that the inverse of $\boldsymbol{M}$ approximately accounts for multiple interactions, yielding a nonlinear algorithm in $\varepsilon_{t}(\boldsymbol{r})-\varepsilon_{b}(\boldsymbol{r})$. Note that the aforementioned property of $\boldsymbol{G}\left(\boldsymbol{r}, \boldsymbol{r}^{\prime}\right)$ driving the extended-Born solution (namely, that $G\left(\boldsymbol{r}, \boldsymbol{r}^{\prime}\right)$ is large for $\boldsymbol{r}$ in the vicinity of $\left.\boldsymbol{r}^{\prime}\right)$ is true for general Green's functions, including that for the layered-media considered here. Moreover, the approximation is enhanced if the medium in which $\boldsymbol{r}^{\prime}$ resides is lossy, such as for a target buried in lossy soil.

If the integral term in (5) is ignored, extended-Born reduces to the classical Born approximation. Moreover, the extended-Born approximation involves the inversion of a $3 \times 3$ matrix $\boldsymbol{M}(\boldsymbol{r})$ for each $\boldsymbol{r} \in V$. These matrices are computed by representing $\varepsilon_{t}(\boldsymbol{r})-\varepsilon_{b}(\boldsymbol{r})$ as an aggregate of $N$ three-dimensional (3-D) cubes [16], [17], similar to (2), except now the dielectric inhomogeneity is a scalar, rather than vector electric field. We compute $\boldsymbol{M}(\boldsymbol{r})$ at the $N \boldsymbol{r}_{n}$ that constitute the centers of the cubes. Consequently, we need to invert $N 3 \times 3$ matrices, constituting order $N$ complexity. Since we need only store and then discard a distinct $3 \times 3$ matrix for each of the $N$ points inside the target, 
memory requirements of extended Born are also substantially less than those of the MoM.

\section{Green's Function Evaluation}

As described in [12], [13], each term in the dyadic Green's function can be expressed in terms of functions of the form

$$
S_{n}\left[f\left(k_{\rho} ; z, z^{\prime}\right)\right]=\int_{0}^{\infty} f\left(k_{\rho} ; z, z^{\prime}\right) J_{n}\left(k_{\rho} \rho\right) k_{\rho}^{n+1} d k_{\rho}
$$

where the path of integration is the traditional Sommerfeld contour [12], [13]. Each dyadic component generally consists of a "direct" term, corresponding to source radiation in a homogeneous medium, plus a "reflection" term, corresponding to interaction with the layer boundaries. Each direct term is integrated in closed form using the Sommerfeld or Weyl identity [23]

$$
\frac{e^{-j k r}}{r}=-j \int_{0}^{\infty} d k_{\rho} k_{\rho} J_{o}\left(k_{\rho} \rho\right) \frac{\exp \left(-j \sqrt{k^{2}-k_{\rho}^{2}}|z|\right)}{\sqrt{k^{2}-k_{\rho}^{2}}} .
$$

A useful tool for integration of the remaining "reflection" term is the complex image technique [2], [14], [15]. While this has proven a very powerful tool for radar applications [2], [15], we have found it much less reliable for the EMI frequencies $(\mathrm{kHz})$ of interest here.

To circumvent these difficulties, we have employed a technique related to that discussed in [12]. In particular, note from (6) that the (generally complicated) functions $f\left(k_{\rho} ; z, z^{\prime}\right)$ are independent of the radial distance $\rho$, the latter accounted for by the Bessel function. We therefore precompute the required $f\left(k_{\rho} ; z, z^{\prime}\right)$ for the "reflection" term of each dyadic component for the range of $z$ and $z^{\prime}$ of interest. This tabulation is performed along a path in the complex $k_{\rho}$ plane [24], along which the $f\left(k_{\rho} ; z, z^{\prime}\right)$ are generally slowly varying (see Fig. 1), thereby reducing the number of discrete complex $k_{\rho}$ that need be considered to accurately represent $f\left(k_{\rho} ; z, z^{\prime}\right)$. This deformed path of integration therefore reduces the required spectral-domain tabulation. The spectral-domain table thus established, we now evaluate the requisite integrals of the form in (6) for the range of $\rho$ required (dictated by the target size). We thereby generate a table of the dyadic Green's function components in the $\left(z, z^{\prime}, \rho\right)$ domain, discarding the $f\left(k_{\rho} ; z, z^{\prime}\right)$ one-by-one, as they are no longer needed. Dividing Green's function tabulation into a separate, initial spectral-domain computation, followed by conversion to the space domain, results in significant savings, for we need only calculate the requisite $f\left(k_{\rho} ; z, z^{\prime}\right)$ once for a given $z$ and $z^{\prime}$ (for all $\rho$ ). Interpolation in the $\left(z, z^{\prime}, \rho\right.$ ) space is performed as in [12].

\section{EXAMPLE RESULTS}

\section{A. Preliminaries}

All computations are performed using parameters characteristic of an actual wideband EMI sensor, termed the GEM-3 [7]. The parameters of the GEM-3 are detailed in Fig. 2. The two excitation loops are designed with radii and currents such that the normal component of the magnetic field vanishes along the

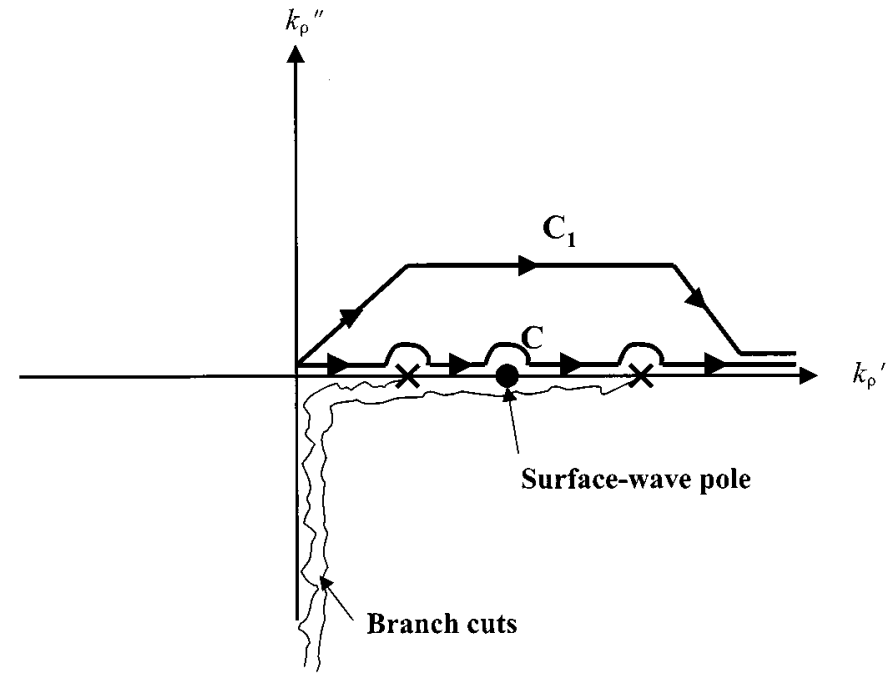

Fig. 1. Schematization of the path of integration in the complex $k_{\rho}$ plane $\left(k_{\rho}=k_{\rho}^{\prime}+j k_{\rho}^{\prime \prime}\right)$. Contour $C$ is the original path of integration, with consideration of the branch points and surface-wave poles (for layered medium). Contour $C_{1}$ is chosen such that the poles and branch points are avoided, and the variation of the spectral domain Green's function is relatively smooth. The left branch cut emanates from branch point $k_{o}$ and the right from $k_{o}\left(\varepsilon_{r N}\right)^{1 / 2}$, where $k_{o}$ is the free-space (top layer) wavenumber, and $\varepsilon_{r N}$ is the relative dielectric constant of the bottom-most layer in a multilayered environment.

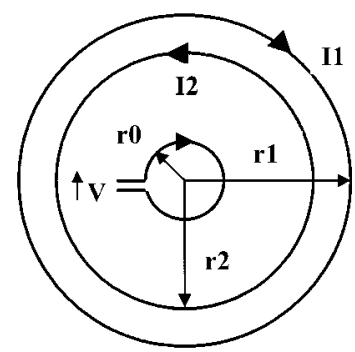

Fig. 2. Schematization of the current loops employed by the GEM-3 EMI sensor [7]. The radii are $R 0=4 \mathrm{~cm}, R 1=26 \mathrm{~cm}$, and $R 2=15.84 \mathrm{~cm}$, and the currents are $I 1=10 \mathrm{~A}$ and $I 2=6 \mathrm{~A}$. The loop of radius $R 0$ is wound 600 times.

coil axis (if the sensor is in a vacuum). A third, smaller loop is placed along the sensor axis. This is used to measure the induced voltage. Consequently, the induced voltage is ideally zero unless the sensor is placed in the vicinity of a field-disturbing medium (e.g., a soil half space and possibly a buried target). In the computations presented here, the incident fields due to the two current loops, in the presence of a lossy layered medium, are computed rigorously by integrating the loop currents with the dyadic layered-medium Green's function [12], [13]. Moreover, the induced voltage is computed via Faraday's law as

$$
V=-j \omega \int_{S} \boldsymbol{B} \cdot \boldsymbol{n} d S
$$

where integration is performed over the planar surface $S$ of the inner loop (with unit normal $\boldsymbol{n}$ ). The induced magnetic fields $\boldsymbol{B}$ are computed by integrating the electric fields induced inside the target (computed via MoM or extended-Born) with the dyadic Green's function. In general, the currents on the excitation loops change in the presence of the induced EMI fields (due to the presence of a target), although this relatively small effect is ignored here. 


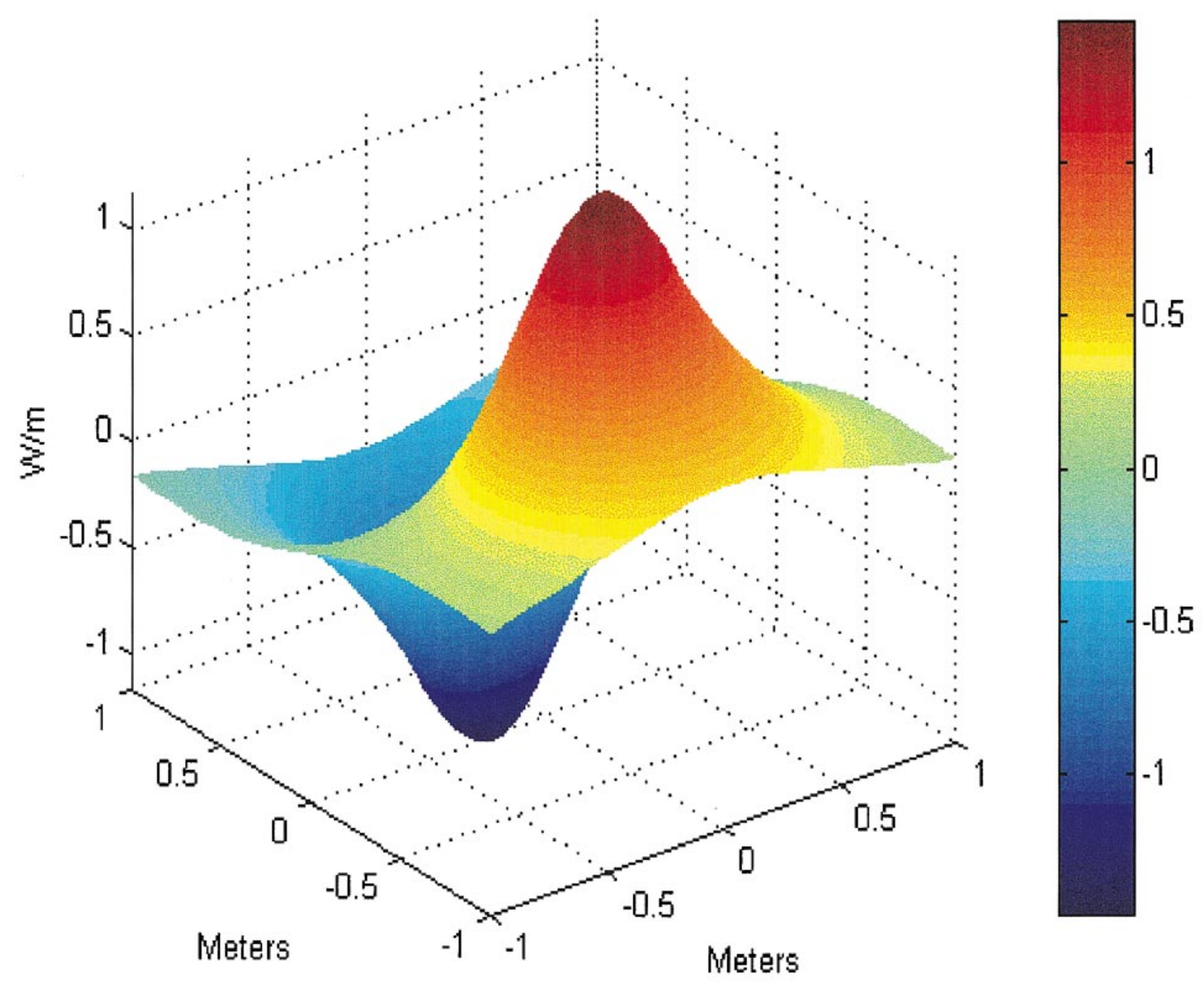

Fig. 3. Space-dependent electric fields $E_{y}(\mathrm{~V} / \mathrm{m}) 10 \mathrm{~cm}$ beneath and parallel to the surface of the GEM-3 sensor. The spatial dimensions are meters, and the imaginary part of $E_{y}$ is plotted. The real part is similar. Note that, as designed, the GEM-3 fields are very small along the sensor axis (origin of this plot).

In the subsequent set of results, we consider several target/soil scenarios, with the induced electric fields inside the target computed by both the extended-Born (approximate) and MoM (reference) methods. The results of the MoM allow quantitative assessment of the extended-Born accuracy for the problem of interest here. Further, the set of examples cover a range of wave phenomenology.

Before proceeding to the results, we note a numerical issue that arose while addressing the computations. It is well known that, for subsectional basis functions, we typically require ten basis functions per wavelength to accurately solve integral equations of the type considered here. For EMI problems, the wavelength is generally much larger than the target dimensions, so this condition on the number of basis functions does not present an issue. From Section II, however, it is also clear that the spatial discritization must be sufficiently fine to accurately represent the fields excited by the EMI sensor $\boldsymbol{E}^{i n c}$ in the absence of a target. As shown in Fig. 2, the GEM-3 has two excitation coils, which generate fast field gradients at positions away from the loop axis. This issue must be considered carefully when dis- cretizing the target. The gradients in $\boldsymbol{E}^{i n c}$ are most significant for shallow targets (near the excitation loops), with such becoming less severe for deeper targets. An example of $\boldsymbol{E}^{i n c}$ at a depth of $10 \mathrm{~cm}$ is shown in Fig. 3 (at $500 \mathrm{KHz}$ ). In the results presented here, we systematically increased the number of subsectional basis functions until numerical convergence was achieved.

\section{B. Single Target}

In our first set of results, we consider the GEM-3 response due to a fixed buried target as a function of the soil properties and the EMI frequency. With regard to the soil properties, measurements have been performed for soil samples taken from the Yuma Proving Ground, Yuma, AZ [1]. These measurements are extrapolated to the EMI frequencies of interest here. We have found that the frequency-dependent relative permittivity is well modeled by $\varepsilon_{r}=\varepsilon^{\prime}-j \varepsilon^{\prime \prime}-j \sigma / \omega \varepsilon_{o}$, where $\varepsilon^{\prime}$ and $\varepsilon^{\prime \prime}$ are constants, and $\sigma$ is the conductivity $(\mathrm{S} / \mathrm{m})$. The conductivity term accounts for the frequency dependence of the complex $\varepsilon_{r}$. The 
TABLE I

SOIL PROPERTIES EXTRAPOLATED From DATA IN [1] FOR SOIL From Yuma, AZ. THE RELATIVE Dielectric CONSTANT IS EXPRESSED AS $\varepsilon_{r}=\varepsilon_{r}^{\prime}-j \varepsilon_{r}^{\prime \prime}-j \sigma / \omega \varepsilon_{o}$, WHERE $\varepsilon_{r}^{\prime}$ AND $\varepsilon_{r}^{\prime \prime}$ ARE CONSTANTS AND $\sigma$ IS THE CONDUCTIVITY (S/m). THE ANGULAR FREQUENCY IS REPRESENTED BY $\omega$. The Data is Tabulated as a Function of the Soil Moisture Content, By PERCENTAGE WeIGHT

\begin{tabular}{c|c|c|c} 
Water Content & $\epsilon_{\mathrm{r}}{ }^{\prime \prime}$ & $\epsilon_{\mathrm{r}}{ }^{\prime \prime}$ & $\sigma(\mathrm{S} / \mathrm{m})$ \\
\hline $5 \%$ & 5.0 & 0.2 & 0.01 \\
\hline $10 \%$ & 8.5 & 0.5 & 0.03 \\
\hline $15 \%$ & 14.5 & 0.9 & 0.06 \\
\hline $20 \%$ & 24 & 1.5 & 0.1 \\
\hline
\end{tabular}

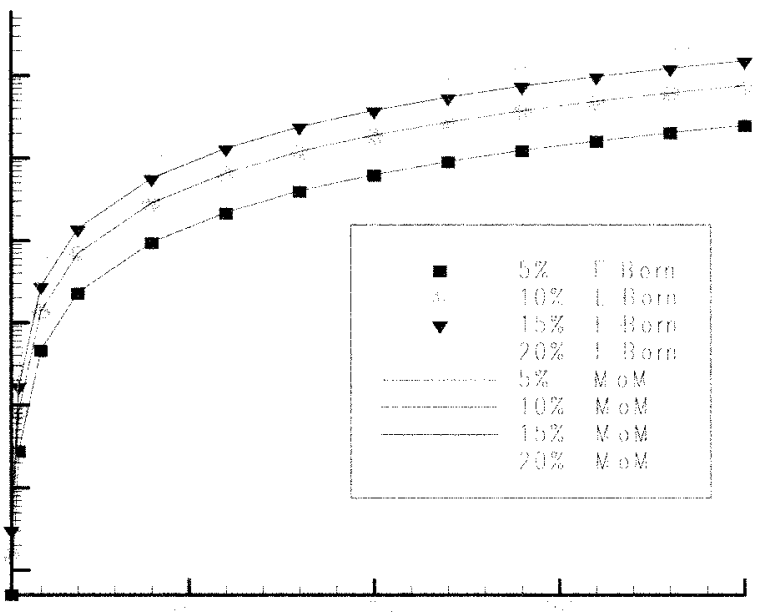

Fig. 4. Magnitude of the voltage induced by a lossless cube target with $16 \mathrm{~cm}$ sides and dielectric constant $\varepsilon_{r}=2.0$, buried to a depth of $12 \mathrm{~cm}$ between the air-ground interface and the target's top surface. The GEM-3 coils are parallel to the soil interface at a height of $4 \mathrm{~cm}$ above the soil, with the sensor axis oriented through the target center. Results are plotted for various soil types, distinguished by the percentage of water content by weight (see Table I). The curves represent the MoM solution, and the points are the results of the extended-Born method.

$\varepsilon^{\prime}, \varepsilon^{\prime \prime}$ and $\sigma$ used here are tabulated in Table I as a function of the soil moisture content (percentage water by weight).

In Fig. 4, we plot the frequency-dependent GEM-3 response due to a lossless cube target of $16 \mathrm{~cm}$ sides and dielectric constant $\varepsilon_{r}=2.0$, buried to a depth of $12 \mathrm{~cm}$ between the airground interface and the target's top surface. The GEM-3 coils are parallel to the soil interface, at a height of $4 \mathrm{~cm}$ above the soil, with the sensor axis oriented through the target center. In Fig. 4, the curves represent the results of the MoM, where the points denote computations from the extended-Born method. The results in Fig. 4 are for the magnitude of the voltage. For these results, the imaginary component of the voltage is two orders of magnitude below its real counterpart. The three principal observations from Fig. 4 are the following.

1) The induced voltage increases with EMI frequency.

2) The extended-Born method yields results that are in close accord with the reference MoM results.

3) The increased target-soil contrast with increasing water content yields the expected increase in the target signature.
Note that at $20 \%$ water content (see Table I), there is a substantial contrast between the target and soil, but the extended-Born method still yields quite accurate results. The extended-Born solutions are also significantly more efficient than the MoM computations. For these computations, we utilized $N=512$ cubes (1536 unknowns). For computation of the electric fields induced inside the target, less than 1 minute was required for the extended-Born solution, while greater than 12 min were required for the MoM computations. All computations reported here were performed on a typical Pentium II personal computer. For both the MoM and extended-Born solutions, we precomputed the dyadic Green's function table (see Section II-C), and this required approximately $5 \mathrm{~min}$ of CPU time on a personal computer.

For classification of the subsurface target, the spatial variation of the EMI signature is also of importance. In Fig. 5(a) and (b), we plot the space-dependent EMI signature of the GEM-3 sensor at $10 \mathrm{kHz}$ for target depths of 25 and $45 \mathrm{~cm}$. The sensor coils are parallel to the air-ground interface, at a height of 2 $\mathrm{cm}$ above the soil. For this example, we consider a lossless spherical target of $10 \mathrm{~cm}$ diameter and $\varepsilon_{r}=3.5$, and the target depth is defined relative to the target center. As before, the solid curves represent the MoM results, while the points denote the extended-Born solution. These results were computed using $N=257$ cubes, and the relative computational requirements of the MoM and extended-Born results were similar to those required for Fig. 4. The voltage magnitude is plotted, and again, the imaginary part of the voltage is two orders of magnitude smaller than that of the real part. For the target considered here, we note that the maximum signal response does not occur when the sensor axis is aligned with the target center. This is attributed to the details of the fields emitted by the sensor (see Fig. 2). In particular, the maximum excitation fields $\boldsymbol{E}^{\text {inc }}$ occur slightly away from the sensor center, as dictated by the sensor design (dictated by the goal of achieving a null voltage when the sensor is in a vacuum). Fig. 5 indicates the expected reduction in the sensor response with increased target depth. Moreover, the discrepancies between the MoM and extended-Born solutions are more apparent in the space-dependent results of Fig. 5. In particular, note that the extended-Born error increases as the soil moisture content increases. This is representative of an increased contrast between the target and background. Nevertheless, the extended-Born results faithfully reproduce the principal characteristics of the EMI signature.

\section{Multiple Targets}

The MoM and the extended-Born software are easily adapted to targets of arbitrary shape, including composite targets. In the next set of results, we consider two distinct objects in the sensor's field of view. As indicated in Fig. 6, we consider the GEM-3 response due to proximate square and cylindrical targets, with the sensor operating at $5 \mathrm{MHz}$ (this frequency is chosen arbitrarily). For this example, the complex dielectric constant of the soil $\varepsilon_{r}=\varepsilon^{\prime}-j \varepsilon^{\prime \prime}-j \sigma / \omega \varepsilon_{o}$ is characterized by $\varepsilon^{\prime}=5, \varepsilon^{\prime \prime}=0$, and $\sigma=0.02 \mathrm{~S} / \mathrm{m}$, and both lossless targets have a real dielectric constant $\varepsilon_{r}=2.25$. The space-dependent EMI responses, as computed via MoM and extended-Born, are shown in Fig. 7. We note that the MoM and extended-Born so- 


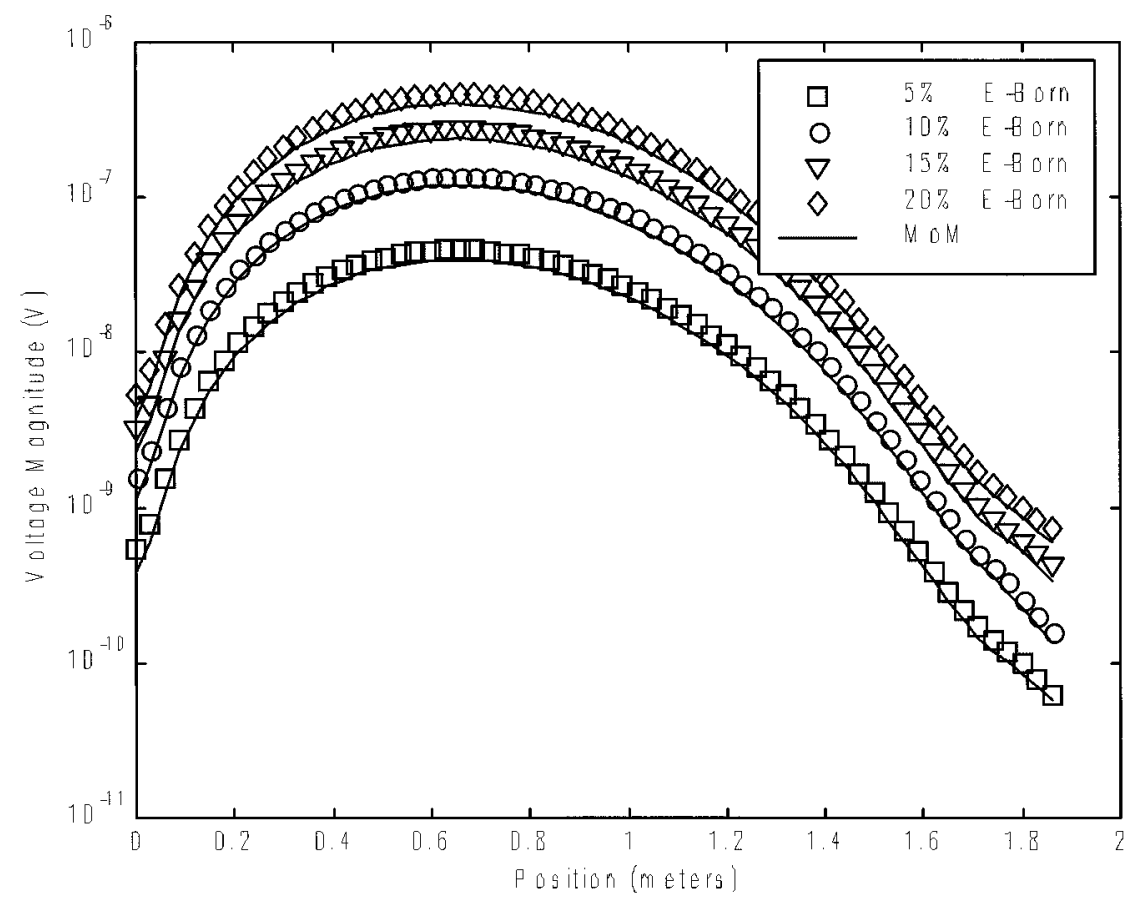

(a)

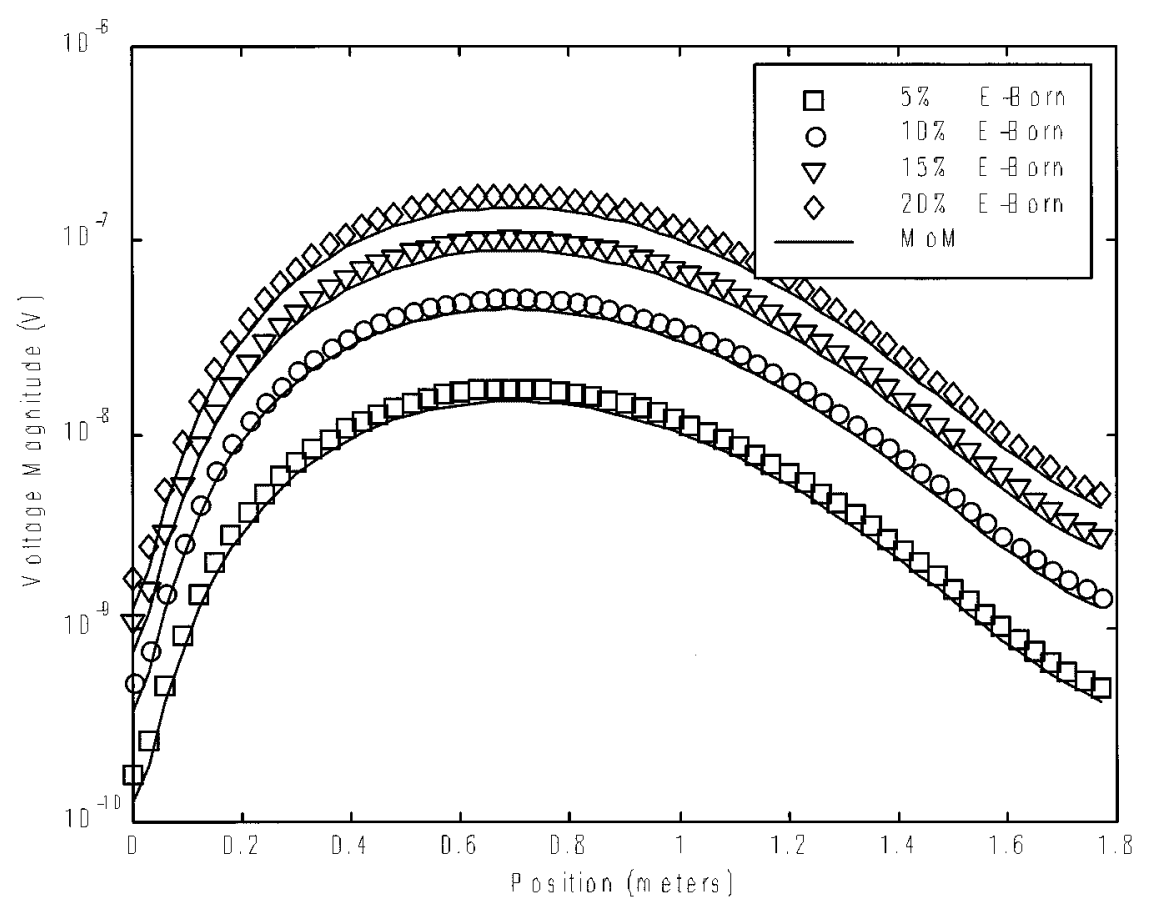

(b)

Fig. 5. Space-dependent EMI signature of the GEM-3 sensor at $10 \mathrm{KHz}$, for a lossless spherical target of $10 \mathrm{~cm}$ diameter and $\varepsilon_{r}=3.5$. The sensor is $2 \mathrm{~cm}$ above the air-ground interface, and the target depth is defined relative to the target center. Results are plotted for various soil types, distinguished by the percentage of water content by weight (see Table I). The curves represent the MoM solution, and the points are the results of the extended-Born method: (a) 25-cm deep target and (b) 45-cm deep target.

lutions are in close agreement. Furthermore, the geometrically larger cylindrical target yields an EMI signature of considerably larger amplitude than the smaller square target. In Fig. 7, we again plot the magnitude of the EMI voltage, the imaginary component of which is again two orders of magnitude smaller than the real component. Similar space-dependent signatures and agreement between MoM and extended-Born solutions was found for all multiple-target tests we considered, although space limitations preclude demonstration of all such examples.

\section{CONCLUSIONS}

A rigorous MoM and approximate extended-Born analysis have been developed for the EMI response of a buried low-loss 


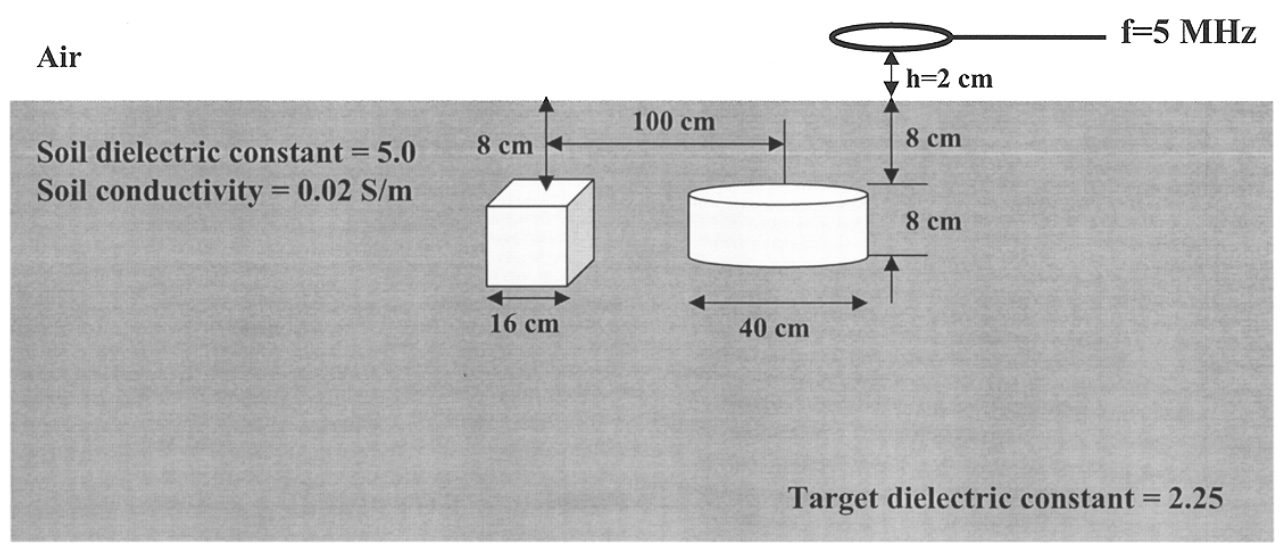

Fig. 6. Schematization of two buried lossless dielectric targets. The sensor is deployed at a height of $2 \mathrm{~cm}$ above the interface at a frequency of $5 \mathrm{MHz}$.

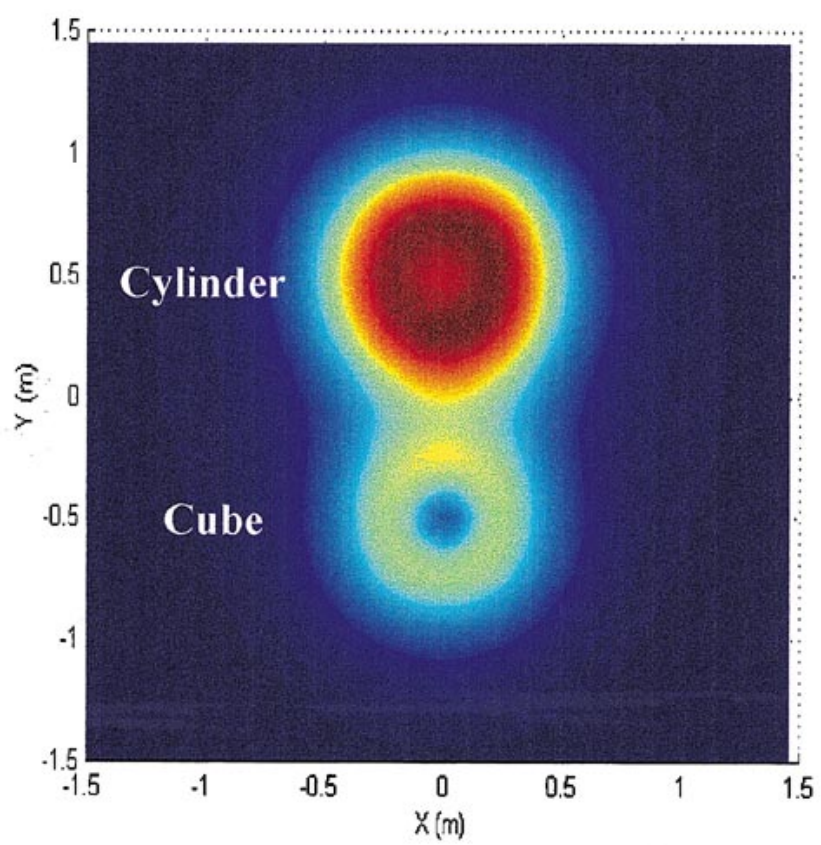

Extended Born

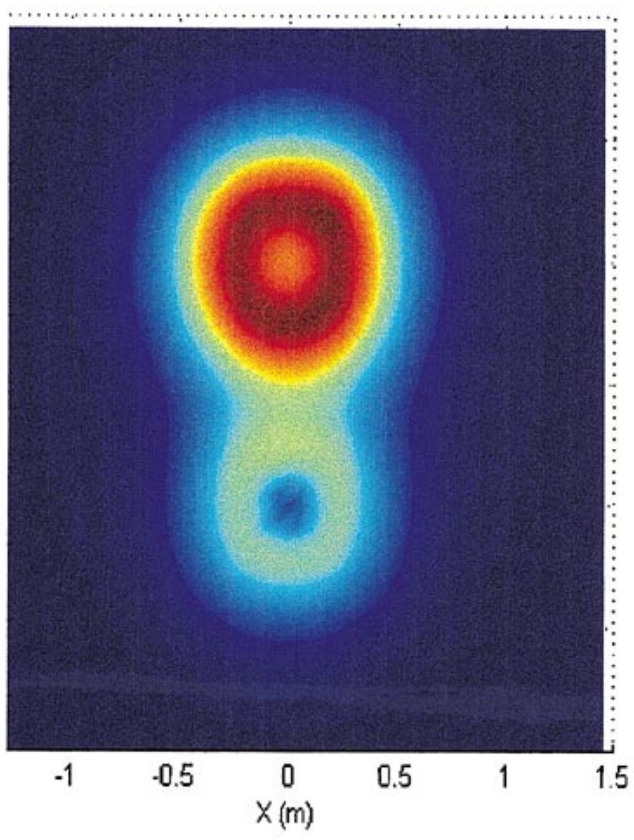

Method of Moments

Fig. 7. Space-dependent induced voltage for the two targets and sensor parameters considered in Fig. 6. The left result is produced by the extended-Born method and the right by MoM.

target embedded in a lossy half space (soil). This research is applicable to many scenarios, in which an EMI sensor may be deployed to sense low-metal-content buried targets (e.g., buried plastic land mines). It has been demonstrated that the highly efficient extended-Born method consistently yields space- and frequency-dependent results that are in close agreement with the reference MoM solution. While the properties of the buried target cannot be changed, those of the soil can. In particular, we have demonstrated the expected strong enhancement of the EMI signature with an increase in the soil water content. It is important to emphasize that this phenomenon (increased water content) is also expected to enhance the electrical contrast for radar applications. However, unlike EMI, the radar excitation is generally severely attenuated as the soil water content increases [1].
We note from Table I that relatively strong electrical contrasts have been considered here and that for such cases, the extended-Born method has been in good agreement with the MoM reference solution. We attribute this accuracy to the loss in the background soil (from Table I for the cases examined, the conductivity increases with increasing soil dielectric constant), which enhances the accuracy of the approximation to $G\left(r, r^{\prime}\right)$, discussed in Section II-B. Nevertheless, extended-Born is an approximation, and it will breakdown as the contrast and target electrical size increases. In this paper, we have sought to consider soil contrasts and target sizes consistent with what may be found in practice, for which the extended-Born method appears to be a particularly attractive solution method.

Having demonstrated the accuracy of the extended-Born algorithm for this problem class, there are several directions for 
future research. For example, we are investigating the use of the extended-Born method in inverse-scattering problems [18]. Furthermore, the extended-Born computed EMI signatures are also appropriate for an optimal Bayesian detection algorithm [1].

\section{REFERENCES}

[1] L. Carin, N. Geng, M. McClure, J. Sichina, and L. Nguyen, "Ultrawide-band synthetic-aperture radar for mine-field detection," IEEE Antennas Propagat. Mag., vol. 41, pp. 18-33, Feb. 1999.

[2] N. Geng and L. Carin, "Wideband electromagnetic scattering from a dielectric body of revolution buried in a layered-medium," IEEE Trans. Antennas Propagat., vol. 47, pp. 610-619, Apr. 1999.

[3] L. Peters Jr., J. J. Daniels, and J. D. Young, "Ground penetrating radar as an environmental sensing tool," Proc. IEEE, vol. 82, pp. 1802-1822, Dec. 1994.

[4] J. M. Bourgeois and G. S. Smith, "A fully three-dimensional simulation of ground penetrating radar: FDTD theory compared with experiment," IEEE Trans. Geosci. Remote Sensing, vol. 34, pp. 28-36, Jan. 1996.

[5] X. X. Zeng and McMechan, "AGPR characterization of buried tanks and pipes,” Geophysics, vol. 62, pp. 797-806, May/June 1997.

[6] A. Witten, I. J. Won, and S. J. Norton, "Subsurface imaging with broadband electromagnetic induction," Inv. Prob., vol. 13, pp. 1621-1639, Dec. 1997.

[7] I. J. Won, D. A. Keiswetter, and D. R. Hanson, "GEM-3: A monostatic broadband electromagnetic induction sensor," J. Environ. Eng. Geophys., vol. 2, pp. 53-64, Mar. 1997.

[8] Y. Das, J. E. McFee, J. Toew, and G. C. Stuart, "Analysis of an electromagnetic induction detector for real-time location of buried objects," IEEE Trans. Geosci. Remote Sensing, vol. 28, pp. 278-288, May 1990.

[9] Y. Das and J.E. McFee, "A simple analysis of the electromagnetic response of buried conducting objects," IEEE Trans. Geosci. Remote Sensing, vol. 29, pp. 342-344, Mar. 1991

[10] N. Geng, C. E. Baum, and L. Carin, "On the low-frequency natural response of conducting and permeable targets," IEEE Trans. Geosci. Remote Sensing, vol. 37, pp. 347-359, Jan. 1999.

[11] M. S. Freund and N. S. Lewis, "A chemically diverse conducting polymer-based electronic nose," in Proc. Nat. Acad. Sci. U.S.A., vol. 92,1995, p. 2652

[12] P. E. Wannamker, G. W. Hohmann, and W. A. SanFilipo, "Electromagnetic modeling of three-dimensional bodies in layered earths using integral equations," Geophys., vol. 49, pp. 60-74, 1984.

[13] K. A. Michalski and D. Zheng, "Electromagnetic scattering and radiation by surfaces of arbitrary shape in layered media-Parts I and II," IEEE Trans. Antennas Propagat., vol. 38, pp. 335-352, March 1990.

[14] S. Vitebskiy, K. Sturgess, and L. Carin, "Short-pulse scattering from buried perfectly conducting bodies of revolution," IEEE Trans. Antennas Propagat., vol. AP-28, pp. 143-151, Feb. 1996.

[15] R. M. Shubair and Y. L. Chow, "A simple and accurate complex image interpretation of vertical antennas present in contiguous dielectric halfspaces," IEEE Trans. Antennas Propagat., vol. 41, pp. 806-812, June 1993.

[16] T. M. Habashy, R. W. Groom, and B. R. Spies, "Beyond the born and Rytov approximations-A nonlinear approach to electromagnetic scattering," J. Geophys. Res. Solid Earth, vol. 98, pp. 1759-1775, Feb. 1993

[17] C. Torres-Verdin and T. M. Habashy, "Rapid 2.5-dimensional forward modeling and inversion via a new nonlinear scattering approximation," Radio Sci., vol. 29, pp. 1051-1079, Jul./Aug. 1994.
[18] E. L. Miller and A. S. Willsky, "Wavelet-based methods for the nonlinear inverse scattering problem using: The extended born approximation," Radio Sci., vol. 31, pp. 51-65, Jan./Feb. 1996.

[19] C. A. Balanis, Advanced Engineering Electromagnetics. New York: Wiley, 1989.

[20] R. F. Harrington, Field Computation by Moment Methods. New York: IEEE Press, 1993.

[21] T. K. Sarkar, X. P. Yang, and E. Arvas, "A limited survey of various conjugate-gradient methods for solving complex matrix equations arising in electromagnetic-wave interactions," Wave Motion, vol. 10, pp. 527-546, Dec. 1988.

[22] T. J. Cui and W. C. Chew, "Fast algorithm for electromagnetic scattering by buried 3-D dielectric objects of large size," IEEE Trans. Geosci. Remote Sensing, vol. 37, pp. 2597-2608, Sept. 1999.

[23] W. C. Chew, Waves and Fields in Inhomogeneous Media. Oxford, U.K.: Oxford Univ. Press, 1996.

[24] K. A. Michalski, "On the efficient evaluation of integrals arising in the Sommerfeld halfspace problem," Proc. Inst. Elect. Eng. F, vol. 132, pp. 312-318, Aug. 1985

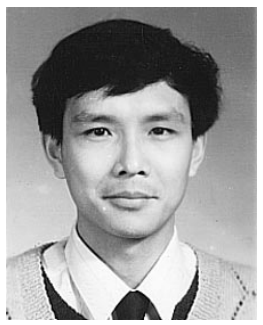

Tiejun Yu (M'99) was born in Jianli, Hubei, China, in 1966. He received the B.S. degree in electrical engineering from the National Defense University of Technology, Changsha, China, in 1988, and the M.S.E. and Ph.D. degrees in electronic engineering,, both from Tsinghua University, Beijing, China, in 1991 and 1996, respectively.

From 1991 to 1996, he was a Lecturer and Assistant Professor at Tsinghua University. From 1997 to 1998, he worked in the Department of Mathematics, University of North Carolina, Charlotte, as a Postdoctoral Researcher. Since then, he has been with the Department of Electrical and Computer Engineering, Duke University, Durham, NC, as a Research Associate. His current research interests include parameter extraction and simulation for VLSI and packaging, electromagnetic scattering, and high-Tc superconducting applications.

Dr. Yu was the winner of the "Best Paper Award" at the 36th Design Automation Conference (DAC), San Francisco, CA, in June 1998.

Lawrence Carin (SM'96) was born on March 25, 1963, in Washington, DC, and received the B.S., M.S., and Ph.D. degrees in electrical engineering from the University of Maryland, College Park, in 1985, 1986, and 1989, respectively.

In 1989, he joined the Electrical Engineering Department, Polytechnic University, Brooklyn, NY, as an Assistant Professor, and became an Associate Professor there in 1994. In September 1995, he joined the Electrical Engineering Department, Duke University, Durham, NC, where he is an Associate Professor. His current research interests include short pulse scattering, subsurface sensing, and wave-based signal processing. He is the Principal Investigator on the U.S Army Multidisciplinary University Research Initiative (MURI) on demining.

Dr. Carin is a member of the Tau Beta Pi and Eta Kappa Nu honor societies. $\mathrm{He}$ is currently an Associate Editor of the IEEE TRANSACTIONS ON ANTENNAS AND PROPAGATION. 